\title{
Conocimientos de los médicos sobre el síndrome de apneas-hipopneas del sueño. ¿Cómo estamos luego de cinco años?
}

\author{
Edmundo Rosales ${ }^{1}$, Martha Egoavil ${ }^{2}$, Claudia La Cruz ${ }^{3}$, Rosemary Figueroa ${ }^{4}$, \\ Jorge Rey de Castro ${ }^{5}$
}

Resumen

Palabras clave

\begin{abstract}
Objetivo: Conocer el nivel de conocimiento sobre el síndrome apneas-hipopneas del sueño de los médicos y contrastarlo con lo informado el año 2001. Diseño: Estudio transversal. Lugar: Cinco congresos realizados los últimos meses del año 2006. Participantes: Asistentes a los congresos. Intervenciones: Cuestionario con 18 preguntas sobre conocimientos del síndrome. Este cuestionario fue aplicado en voluntarios, autoadministrado, único y anónimo. Principales medidas de resultados: Conocimientos sobre el síndrome, su diagnóstico y tratamiento. Resultados: Se recabó 241 cuestionarios. La edad de los participantes fue 36,1 111,7 [20 a 76] años. Conocían lo que era 'apnea del sueño' 237(98\%) y este conocimiento fue adquirido en estudios de pregrado, en 39\%. Un 16\% identificó los riesgos asociados al síndrome; 149(62\%) afirmóque se presentaba exclusivamente en varones obesos y 45(19\%) que era más frecuente en mujeres premenopáusicas; 107 (45\%) no conocía la prevalencia del ronquido en varones mayores de 35 años, 184 (76\%) afirmó que la hipersomnia era el síntoma más frecuente y 94 (39\%) que el insomnio era descrito por pacientes con este síndrome. Ciento ocho (45\%) no indagaban por síntomas relacionados con el síndrome en su práctica cotidiana; 70 (29\%) no reconocieron a la polisomnografía o poligrafía respiratoria como método de diagnóstico; 147 (61\%) identificaron modalidades de tratamiento, pero ninguno identificó a todas ellas; 89(37\%) desconocían que había especialistas que estudian este síndrome en el Perú. Conclusiones: Los médicos saben más sobre esta enfermedad en comparación con lo observado hace 5 años; sin embargo, este conocimiento aún es deficiente. Se debe promover la inclusión de este tema dentro de la educación de médicos y otros profesionales de la salud.
\end{abstract}

Conocimientos, actitudesy prácticas; competencia clínica; síndrome de la apnea del sueño; ronquido.
Physicians' knowledge on obstructive sleep apnea. How are we after five years?

\section{Abstract}

Objective: To determine physicians'knowledge level onobstructive sleep apnea and to compare it with a report published in 2001. Design: Cross-sectional study. Setting: Five congresses developed during 2006. Participants: Congress assitants. Interventions: Questionnaire with 18questions aboutknowledge of the syndrome.

1 Médico-Cirujano. Programa Maestría en Medicina, Universidad Peruana Cayetano Heredia. Lima, Perú.

2 Médico-Cirujano. Programa Maestría en Geriatría y Gerontología, Universidad Peruana Cayetano Heredia. Lima, Perú.

3 Médico-Cirujano. Ex alumno de la Universidad Peruana Cayetano Heredia. Lima, Perú.

4 Estudiante de Medicina. Universidad Peruana Cayetano Heredia. Lima, Perú.

5 Neumología y Trastornos Respiratorios del Sueño. CENTRES y Clínica Anglo Americana. Profesor Principal de la Universidad Peruana Cayetano Heredia. Lima, Perú.
This questionnaire was self administered, unique and anonymous. Main outcome measures: Knowledge of the syndrome, its diagnosis and treatment. Results: Two hundred and forty-one questionnaires were successfully obtained. The age of the participants was $36,1 \pm 11,7$ [20 to 76] years. Two hundred and thirty seven (98\%) knew the meaning of 'sleep apnea', acquired in pre-degree studies in 39\%; $16 \%$ identified disorders associated to the syndrome; 149(62\%) affirmed it appeared exclusively in obese men and 45 (19\%) that it was most frequent in premenopausal women; 107 (45\%) did not know the prevalence of snore in 35 year-old men or older, 184 (76\%) affirmed that hypersomnia was the most frequent symptom and 94 (39\%) that insomnia was described by patients with this syndrome. One hundred and eight (45\%) did not investigate by symptoms related to the syndrome in their daily medical practice; 70 (29\%) did not recognize polysomnography or respiratory polygraphy as a diagnosis method; 147 (61\%) identified treatment modalities but nobody identified all of them; 89(37\%) did not know that there were specialists who study this syndrome in Peru. Conclusions: Physicians know more about 
this disease in comparison with the report done 5 years ago; nevertheless, this knowledge is still deficient. The inclusion of this subject in the education of physicians and other health professionals should be promoted.

Key words: Knowledge, attitudes, practice; clinical competence; sleep apnea syndromes; snoring.

\section{INTRODUCCIÓN}

En el año 2001 se implementó una encuesta en Lima para indagar por el nivel de conocimientos de los médicos sobre el síndrome de apneas-hipoapneas del sueño (SAHS) y se demostró que éste era muy deficiente $\left({ }^{1}\right)$. Han pasado cinco años desde ese estudio y en este periodo se ha implementado tres nuevos laboratorios de sueño, que se añaden a los dos en existencia y tres neumólogos hacen estudios de poligrafía cardiorrespiratoria bajo condiciones no supervisadas, tanto en ambientes hospitalarios como en forma domiciliaria. Así mismo, se ha difundido el tema en numerosas conferencias, mesas redondas, discusión de carteles y resúmenes en el marco de cursos y congresos nacionales. En lo que se refiere a la incorporación al contenido curricular de pregrado, la Universidad Peruana Cayetano Heredia (UPCH) lo hizo por primera vez en el capítulo de enfermedades respiratorias, el año $1998\left(^{2}\right)$, y la Universidad Nacional Mayor de San Marcos (UNMSM) lo ha incorporado en el plan de estudios dentro del capítulo de Neumología $\left({ }^{3}\right)$. De otro lado, autores peruanos han publicado, entre 1999 y 2006, quince artículos en revistas médicas nacionales y cinco en extranjeras, que comprenden contenidos originales y revisiones de temas relacionados al SAHS.

No existe la menor duda, el SAHS produce deterioro neurocognitivo ${ }^{(4-6)}$, daño cardiovascular $\left({ }^{7}\right)$ y cerebrovascular $\left({ }^{8}\right)$. Por otro lado, está plenamente demostrado que se asocia a hipertensión arterial $\left({ }^{9-12}\right)$, enfermedad coronaria $\left({ }^{7,13}\right)$ y cerebrovascular $\left({ }^{8,14-}\right.$ ${ }^{16)}$. Así mismo, está claramente asociado a accidentes de tránsito, debido a la somnolencia $\left({ }^{17-19}\right)$. Finalmente, se ha demostrado que en pacientes con formas severas de enfermedad se asocia a mayor riesgo de morir por infarto al miocardio o accidente cerebrovascular $\left.{ }^{(20-23}\right)$.

Dada la importancia del SAHS, los riesgos asociados y sus consecuencias, nos propusimos como objetivo conocer cuál es el nivel de conocimiento sobre la enfermedad en el medio médico y contrastarlo con el observado el año 2001.

\section{MATERIALES Y MÉTODOS}

La recolección de la información se realizó a través de un cuestionario con 17 preguntas cerradas, de opción múltiple, y una pregunta abierta sobre modalidades de tratamiento. Dicho cuestionario fue utilizado anteriormente en el año 2001 (1) y en esta última ocasión agregamos tres preguntas nuevas. Se incluyó preguntas básicas de filiación y profesión o especialidad. Este cuestionario fue autoadministrado, único y anónimo. La participación fue voluntaria $y$, con el llenado de los cuestionarios, los participantes autorizaron su inclusión en el estudio. El estudio fue revisado y aprobado por el Comité Institucional de Ética de la UPCH, con código 51784.

El cuestionario se aplicó en cinco congresos realizados durante los últimos meses del año 2006: Jornadas Médicas Heredianas 2006, organizado por la UPCH (Lima, 21 a 23 de setiembre), 42 cuestionarios (17\%); I Encuentro Iberolatinoamericano de Asma y EPOC, organizado por la Sociedad Peruana de Neumología (Cuzco, 09 al 11 de octubre), 33 cuestionarios (14\%); XXV Congreso Peruano de Neumología, organizado por la Sociedad Peruana de Neumología (Lima, 12 al 15 de octubre), 156 cuestionarios $(65 \%)$; XIV Congreso Nacional organizado por la Sociedad Peruana de Medicina Interna (Lima, 26 al 29 de octubre), 
7 cuestionarios (3\%); y III Curso Internacional Multidisciplinario, organizado por la Clínica Anglo Americana (Lima, 22 al 24 de noviembre), 3 cuestionarios (1\%). Se recabó 242 cuestionarios; uno presentó inconsistencias insalvables, por lo que fue excluido del análisis, quedando finalmente 241 cuestionarios (Tabla 1).

Todos los datos fueron registrados en una tabla del programa Excel $^{\circledR} \mathrm{V} .11$ y analizados con medidas de tendencia central y en forma porcentual simple, con el programa Epi-Info ${ }^{\circledR}$ v.3.3.2.

\section{RESULTADOS}

La edad media de los participantes fue $36,1 \pm 11,7$ [20 a 76] años; 126 eran mujeres $(52 \%), 111(46 \%)$ hombres y $4(2 \%)$ no respondieron (NR). El año de graduación se muestra en la Figura 1. En cuanto a la profesión de los encuestados, 153 (64\%) fueron médicos generales, 54 (22\%) médicos especialistas, 12 (4\%) médicos residentes, $6(2 \%)$ internos y $16(7 \%)$ estudiantes de medicina. Tres (1\%) no respondieron la pregunta. Entre los especialistas más frecuentes, estuvieron 15 pediatras, 14 neumólogos y 12 internistas. Preguntamos a los participantes en qué universidad se graduaron y se observó que la mayoría era graduado de la UPCH, seguido por la Universidad San Martín de Porres y la UNMSM.

Al preguntar si sabían lo que era el 'apnea del sueño'; 237 (98\%) de los participantes respondieron afirmativamente. El conocimiento relacionado a esta enfermedad había sido adquirido exclusivamente durante sus estudios de pregrado en 93 (39\%), por medio de conferencias o charlas en 50 $(21 \%)$, estudios de posgrado $19(8 \%)$, lectura de revistas científicas o libros $9(4 \%)$ e internet $5(2 \%) ; 60$ encuestados $(25 \%)$ habían adquirido dicho conocimiento de más de una fuente. Tres participantes afirmaron
Tabla 1. Porcentaje de participación de los inscritos a los cinco congresos en donde se realizó el estudio.

\begin{tabular}{|c|c|c|c|}
\hline Cursos & $\begin{array}{l}\text { Número } \\
\text { inscritos* }\end{array}$ & $\begin{array}{l}\text { Número } \\
\text { encuestas }\end{array}$ & $\%$ \\
\hline $\begin{array}{l}\text { - Jornadas Médicas Heredianas } 2006 \\
\text { - XIV Congreso Nacional de }\end{array}$ & 289 & 42 & 14,5 \\
\hline Medicina Interna & 1208 & 7 & 0,6 \\
\hline $\begin{array}{l}\text { - I Encuentro Iberolatinoamericano } \\
\text { de Asma y EPOC }\end{array}$ & 399 & 33 & 8,3 \\
\hline $\begin{array}{l}\text { - XXV Congreso Peruano de } \\
\text { Neumología } 1\end{array}$ & 171 & 156 & 132 \\
\hline - III Curso Internacional & & & \\
\hline Multidisciplinario & 275 & 3 & 1,1 \\
\hline Total & 3342 & 241 & 7,2 \\
\hline
\end{tabular}

que lo habían hecho a través de familiares que sufrían la enfermedad o conversando con otros profesionales en su centro de trabajo, que conocen del tema. Un participante $\operatorname{NR}(0,5 \%)$.

Al indagarse por los riesgos asociados al SAHS -como hipertensión arterial, accidente cerebrovascular, enfermedad coronaria, accidentes de tránsito o mayor riesgo de morir-, $39(16 \%)$ identificaron las cinco condiciones, $18(8 \%)$ cuatro, $42(17 \%)$ tres, $60(25 \%)$ dos y $68(28 \%)$ solo una. El factor de riesgo asociado a SAHS identificado con mayor frecuencia fue la hipertensión

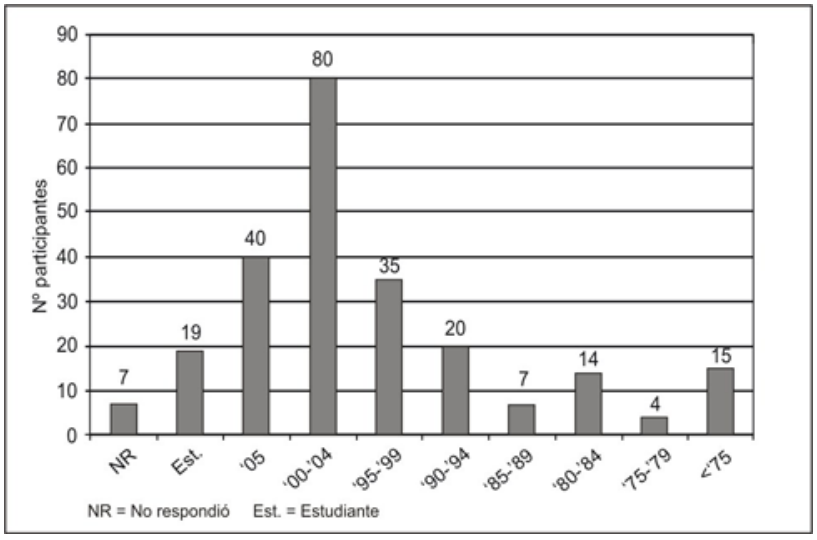

Figura 1. Distribución de los participantes por año de graduación. 
arterial, en $134(56 \%)$ encuestados. Catorce $(6 \%)$ NR.

Ciento cuarenta y nueve (62\%) afirmaron que el SAHS se presenta exclusivamente en varones obesos y $6(2 \%)$ NR. Cuarenta y cinco (19\%) afirmaron que el SAHS es más frecuente en mujeres premenopáusicas, 9 (4\%) participantes NR. Con relación a la prevalencia del ronquido en varones por encima de los 35 años, 6 (3\%) contestaron que era $5 \%, 26(11 \%) 10 \%, 36(15 \%) 20 \%, 134$ $(55 \%)$ entre 30 y $50 \%$ y $16(7 \%)$ que dicha prevalencia era $75 \%$. Veintitrés (9\%) NR.

Ciento ochenta y cuatro $(76 \%)$ afirmaron que la hipersomnia es el síntoma más frecuente del SAHS, 94 (39\%) que el insomnio es síntoma descrito por pacientes con SAHS y $108(45 \%)$ que durante su práctica cotidiana no indagan en sus pacientes por síntomas como ronquido nocturno, pausas durante el dormir e hipersomnia.

Con relación a los métodos para documentar el diagnóstico de enfermedad (Figura 2), $148(61 \%)$ respondieron polisomnografía (PSG) o poligrafía respiratoria (PR) y 23 (10\%) PSG o PR en combinación con otras alternativas, como interrogatorio a la pareja del paciente, uso de cuestionarios con escalas de ronquido y/o hipersomnia, infor-

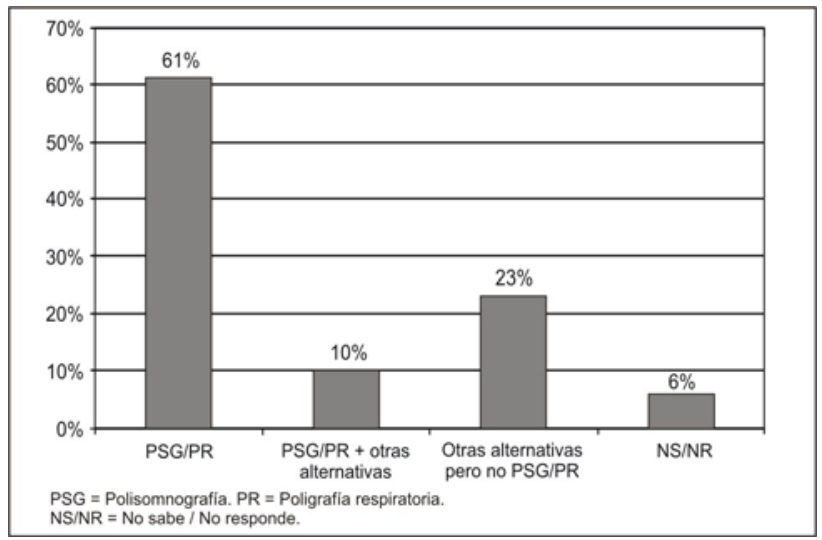

Figura 2. Distribución de las respuestas sobre métodos para diagnosticar el síndrome apnea-hipopneas (SAHS). mación clínica y oximetría de pulso nocturna. El resto $(23 \%)$ proporcionó respuestas que no incluían PSG o PR. Catorce (6\%) NR.

Sobre las modalidades de tratamiento empleadas en el SAHS, 147 (61\%) respondieron la pregunta; $14(9,5 \%)$ describieron tratamientos no convencionales sin sustento en la literatura médica, como el empleo de antihistamínicos, broncodilatadores, teofilina, oxigenoterapia nocturna, psicoterapia o dormir sentado. De otro lado, 133 identificaron modalidades de tratamiento aceptadas por la literatura médica. Así, 91 (68\%) mencionó una modalidad, 30 (23\%) dos modalidades, 10 (8\%) tres modalidades y 2 (1\%) cuatro modalidades. La modalidad terapéutica más mencionada entre los que respondieron a la pregunta fue (Figura 3) la presión positiva continua sobre la vía aérea o CPAP, en $91(62 \%)$. En el grupo que mencionó solo una modalidad terapéutica, las respuestas fueron CPAP o BiPAP, en 59 (65\%), seguido por control de peso, $18(20 \%)$, procedimientos quirúrgicos, $7(8 \%)$, terapia posicional al dormir, $6(6 \%)$, y 1 (1\%), dispositivos orales.

Finalmente, 89 (37\%) participantes desconocían que en el Perú había especialistas que estudian a pacientes con SAHS, 7 (3\%) NR.

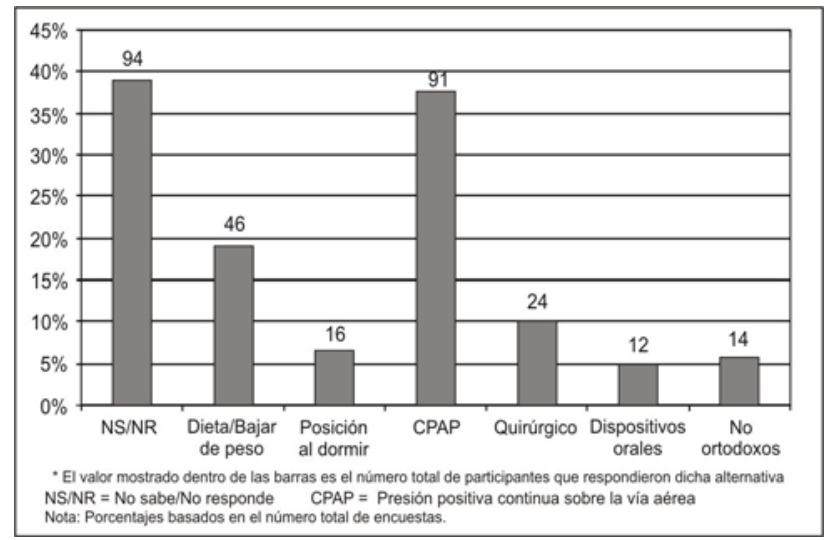

Figura 3. Distribución de las respuestas sobre tratamiento para el síndrome apnea-hipopneas (SAHS). 


\section{DISCUSIÓN}

El SAHS es el desorden médico más frecuentemente asociado a hipersomnia o somnolencia. Afecta a casi 2 a $4 \%$ de mujeres y 4 a $6 \%$ de varones $\left({ }^{24-27}\right)$ de la población general de edades medias de la vida, observándose que la prevalencia aumenta claramente con la edad $\left({ }^{28}\right)$.

El SAHS se define como un trastorno respiratorio del sueño asociado con síntomas diurnos y nocturnos e implica episodios repetidos de colapso parcial o total de la vía aérea superior, que se presentan durante el sueño, provocando finalmente interrupción y fraccionamiento del mismo, produciendo excesiva somnolencia diurna $\left({ }^{29-32}\right)$.

El tema de los trastornos del sueño sigue siendo algo relativamente nuevo en nuestro medio y aunque es muy específico y de dominio del especialista, debe ser del conocimiento de médicos generales y otros especialistas, debido a la asociación que existe entre el SAHS y otras patologías, como hipertensión arterial refractaria ${ }^{(33}$ ) o insuficiencia cardiaca $\left({ }^{34}\right)$. Esto no solo involucra a médicos, sino también a otros profesionales de la salud $\left({ }^{35}\right)$. Aunque está demostrado que el tratamiento modifica la mortalidad, morbilidad y calidad de vida de los pacientes $\left({ }^{36-38}\right)$, la mayoría de los casos permanece sin una terapia adecuada, como consecuencia de un subdiagnóstico $\left({ }^{39,40}\right)$.

En los EE UU, el costo que conlleva al sistema de salud que el SAHS no sea diagnosticado o tratado llega a la suma de 3,4 miles de millones de dólares $\left({ }^{41}\right)$. En un estudio hecho por Ayas y col $\left({ }^{42}\right)$, en pacientes con SAHS moderado a severo que recibieron tratamiento con CPAP, encontraron que la calidad de vida y los accidentes de tránsito con este tratamiento fueron costoeficaces.

En el estudio realizado en Perú, en el $2001\left({ }^{1}\right)$, se observó un deficiente conoci- miento de los médicos con relación al SAHS. El sueño había sido siempre considerado un evento básicamente anecdótico y fue por ello que los programas de educación universitaria en los niveles de pregrado y segunda especialización de las diversas facultades de medicina no incluían sistemáticamente este contenido dentro de su programa curricular. Nosotros esperamos que esa realidad haya cambiado en el transcurso de los últimos cinco años.

Como se ve en los resultados, el cuestionario fue contestado en su mayoría por médicos generales graduados en los últimos cinco años, algo muy similar a lo observado en la encuesta del 2001. Esto es algo esperado, ya que los médicos recién graduados están particularmente interesados en acumular créditos académicos para su currículum vitae y ulteriores estudios de posgrado.

Solo una pequeña fracción no conocía o no respondió sobre el conocimiento de la apnea del sueño. Esta cifra fue diez veces menor a la encontrada el año 2001. En nuestro estudio, menos de la mitad adquirió dichos conocimientos en la universidad (pregrado y posgrado), siendo ligeramente superior al 2001.

Otro resultado resaltante está relacionado al hecho de que casi la quinta parte de los participantes adquirió sus conocimientos exclusivamente a través de conferencias o charlas, superando a otras fuentes, como la lectura de revistas científicas, libros o internet. Llamó nuestra atención el escaso uso de una herramienta tan importante, como internet. En el 2001, la lectura de revistas científicas fue la fuente de información más frecuente, luego de los estudios universitarios. Esto es algo reconfortante, ya que indicaría que de alguna manera las sociedades o instituciones académicas que organizan los congresos, cursos o charlas dirigidos a los profesionales de la salud están tomando mayor interés en incluir el tema en el contenido de sus acti- 
vidades programadas. Pero, cabe recalcar que de los cinco congresos en donde se aplicó el cuestionario, solo en dos el tema de trastornos del sueño formó parte de la temática (Congreso Peruano de Neumología y el Curso Internacional Multidisciplinario).

Casi todos los participantes identificaron uno o más factores de riesgo asociados al SAHS; y considerando solo a las personas que respondieron la pregunta, cerca de un tercio identificó un factor de riesgo y casi una quinta parte señaló los cinco factores. El identificado con más frecuencia fue el riesgo de morir. A diferencia del 2001, el porcentaje de personas que no respondió o no sabía disminuyó en casi dos tercios.

Las tres quintas partes de los participantes afirmaron que el SAHS está asociado a personas obesas de sexo masculino, una cifra mayor que en el 2001. Al respecto, estudios epidemiológicos basados en población general han estimado que el riesgo en los varones es 2 a 3 veces mayor, comparado con las mujeres $\left({ }^{24}\right)$. Además, hay una clara asociación entre el peso y SAHS $\left({ }^{43}\right)$. Se ha visto que un incremento de $10 \%$ del peso corporal lleva a un incremento de seis veces en el riesgo a desarrollar SAHS $\left({ }^{44}\right)$. Pero, debe tenerse en cuenta que el SAHS no es exclusivo de este tipo de población $\left({ }^{45}\right)$.

A diferencia del 2001, en esta oportunidad se preguntó si el SAHS era frecuente en mujeres premenopáusicas. En lo que respecta a esto, Young y col. $\left({ }^{46}\right)$ encontraron que la transición hacia la menopausia conlleva a un incremento en la probabilidad de tener un trastorno respiratorio del sueño. También Bixler y col. $\left({ }^{24}\right)$ observaron que la prevalencia de mujeres premenopáusicas era menor que las posmenopáusicas $(0,6 \%$ vs. 2,7\%); pero, además observó que la prevalencia de ambos grupos de mujeres era menor que en los hombres $(3,9 \%)$. Basado en esta información, el $23 \%$ respondió incorrectamente a dicha pregunta.
Los síntomas más comunes referidos por el paciente con SAHS o las observaciones hechas por la pareja o compañero/a de cuarto con sospecha de SAHS fueron ronquido, jadeo o resoplidos, pausas respiratorias y excesiva somnolencia diurna (ESD) o hipersomnia $\left({ }^{31}\right)$. El ronquido es el síntoma nocturno más frecuente y puede ser identificado con facilidad $\left({ }^{31,32}\right)$. Estudios epidemiológicos en población general informan una prevalencia de ronquido entre 18 y $55 \%$ en hombres y entre 15 y $36 \%$ en mujeres $\left({ }^{47-50}\right)$. En el Perú, Rey de Castro y col. $\left({ }^{50}\right)$ encontraron que $27 \%$ de varones y $18 \%$ de mujeres son roncadores. La mitad de los participantes respondieron correctamente que la prevalencia de ronquido en varones era entre 30 a $50 \%$.

La ESD es causada por despertares frecuentes provocados por eventos respiratorios obstructivos, lo que deriva en fragmentación del sueño $\left({ }^{47}\right)$. La ESD es un síntoma común en pacientes con SAHS, pero de menor frecuencia que el ronquido $\left({ }^{31}\right)$. Es importante recalcar que 30 a $50 \%$ de personas sin diagnóstico de SAHS tienen ESD y además muchos pacientes con SAHS refieren no sufrir de ESD $\left({ }^{29,47}\right)$. Aproximadamente, las tres cuartas partes de los participantes afirmaron erróneamente que la hipersomnia era el síntoma más frecuente en pacientes con SAHS. Así mismo, más de la mitad negó que el insomnio sea un síntoma descrito por dichos pacientes. El insomnio se presenta particularmente en mujeres y personas ancianas con SAHS $\left({ }^{31,51,52}\right)$, en quienes se ha descrito insomnio intermedio y el levantarse más temprano de lo deseado. Por lo tanto, también es importante considerar al SAHS como causa de insomnio.

Aproximadamente, la mitad de participantes no indagaba por síntomas, como ronquido, pausas respiratorias o somnolencia anormal en su práctica médica cotidiana. Esta proporción llegó a ser inferior en la encuesta del 2001, aproximadamente las tres quintas partes de lo obtenido en nuestro es- 
tudio. Este hallazgo es preocupante, ya que no es posible plantear un nivel inicial de sospecha diagnóstica si no se pregunta por esta sintomatología cardinal. Los estudios demuestran que aproximadamente $93 \%$ de mujeres y $82 \%$ de hombres con SAHS de grado moderado a severo no han sido identificados $\left(^{53}\right)$, siendo generalmente el médico de atención primaria el primero en ver a este tipo de pacientes $\left({ }^{54}\right)$.

Las guías o consensos, tanto europeos $\left({ }^{32}\right)$, americanos $\left({ }^{55}\right)$ como peruanos $\left({ }^{56,57}\right)$, consideran a la polisomnografía (PSG) como el estándar dorado para el diagnóstico del SAHS. Debido a la gran demanda que existe para realizar PSG, la complejidad para implementarla y el elevado costo de la prueba, en los últimos años están apareciendo equipos simplificados que sacrifican las variables neurofuncionales de la polisomnografía y conservan los sensores orientados al registro cardiorrespiratorio. Estas pruebas son las llamadas poligrafías respiratorias (PR). Las tres cuartas partes afirmaron que la PSG o la PR es el método para documentar el diagnóstico de SAHS, a diferencia de la mitad que así lo afirmó el año 2001.

El tratamiento del SAHS tiene como objetivo resolver los signos y síntomas de la enfermedad, corregir el índice de apneahipopnea, desaturación de hemoglobina y normalizar la calidad del sueño $\left({ }^{31,32}\right)$; asimismo, proteger al paciente de los factores de riesgo asociados. Las medidas generales son higiene del sueño, control de la obesidad, evitar el alcohol, tabaco y finalmente fármacos que agravan la enfermedad, como sedantes, tranquilizantes e hipnóticos. Otros tratamientos son los dispositivos orales durante el dormir, el tratamiento con CPAP y la cirugía de la vía aérea alta. Ningún encuestado identificó todas las modalidades convencionales de tratamiento y en la encuesta del 2001 solo una persona lo hizo. Dos personas reconocieron cuatro modalidades de tratamiento aceptadas por la literatura médica. Más de un tercio de los partici- pantes no respondió o no conocía las modalidades de tratamiento; esta cifra es la mitad de lo registrado el 2001. Aproximadamente, las dos terceras partes identificaron al CPAP como modalidad de tratamiento del SAHS.

Un poco menos de dos tercios de participantes sabía de la existencia de médicos especialistas en sueño en el Perú; esta pregunta no fue formulada el año 2001.

Nuestro estudio tiene algunas limitaciones. Los resultados obtenidos de los cuestionarios se basan en un autorreporte sustentado en la apreciación del encuestado; ésta es una limitación conocida de estas herramientas. También, tiene limitaciones inherentes a la recopilación de información por medio de cuestionarios, como el sesgo de memoria. Finalmente, la escasa participación de los asistentes a los eventos se hizo evidente a lo largo del estudio. Esta no es por lo tanto una población representativa de los médicos que asistieron a los eventos antes mencionados y nuestros resultados podrían estar sub o sobrestimados. Es muy preocupante el escaso interés de los médicos (Tabla 1) por participar en estudios de esta naturaleza, que por cierto proporcionan información muy útil.

En líneas generales y tomando en cuenta las limitaciones se podría concluir que los profesionales médicos saben más sobre esta enfermedad, su forma de diagnóstico y su tratamiento en comparación con lo encontrado hace 5 años. Persiste un nivel de deficiencia en el conocimiento sobre identificación de riesgos y propuestas terapéuticas. No se ha conseguido un cambio notorio en la actitud de los médicos para que indaguen por los síntomas cardinales del SAHS en su práctica clínica cotidiana. Debe hacerse todos los esfuerzos necesarios para difundir el tema, promover su contenido en los planes de estudios de pre y posgrado o segunda especialización, así como en cursos o congresos dirigidos a los profesionales de la salud. Es indispensable hacer in- 
vestigación en las diferentes facetas que ofrecen los trastornos respiratorios del sueño. Considerando el daño que produce la enfermedad, la difusión de esta información debe ser considerada una prioridad.

\section{REFERENCIAS BIBLIOGRÁFICAS}

1. Rey de Castro J, Hernández J. ¿Cuánto saben los médicos sobre el síndrome apnea hipopnea obstructiva del sueño? Rev Soc Peru Med Interna. 2001;14:17-22.

2. Universidad Peruana Cayetano Heredia. Facultad de Medicina Alberto Hurtado. Departamentos Académicos de Cirugía, Medicina, Patología y Radiología. Syllabus Clínica Médica 1. Capítulo integrado de Respiratorio NID-2550. Cuarto año de Medicina. Lima: UPCH; 1998.

3. Universidad Nacional Mayor de San Marcos. Facultad de Medicina Humana. Escuela Académica Profesional de Medicina Humana. Syllabus de Medicina Interna. Año Académico 2004 (Código MH0440). Lima: UNMSM; 2007.

4. Quan SF, Wright R, Baldwin CM, Kaemingk KL, Goodwin JL, Kuo TF, et al. Obstructive sleep apnea-hypopnea and neurocognitive functioning in the Sleep Heart Health Study. Sleep Med. 2006;7:498-507.

5. Engleman HM, Douglas NJ. Sleep - 4: Sleepiness, cognitive function, and quality of life in obstructive sleep apnoea/ hypopnoea syndrome. Thorax. 2004;59:618-22.

6. Schneider C, Fulda S, Schulz H. Daytime variation in performance and tiredness/sleepiness ratings in patients with insomnia, narcolepsy, sleep apnea and normal controls. J Sleep Res. 2004;13:373-83.

7. Budhiraja R, Quan SF. Sleep-disordered breathing and cardiovascular health. Curr Opin Pulm Med. 2005;11:501-6.

8. Hermann DM, Bassetti CL. Sleep-disordered breathing and stroke. Current Opinion in Neurology. 2003;16:87-90.

9. Robinson GV, Stradling JR, Davies RJO. Sleep - 6: Obstructive sleep apnoea/hypopnoea syndrome and hypertension. Thorax. 2004;59:1089-94.

10. Sharabia Y, Daganb Y, Grossman E. Sleep apnea as a risk factor for hypertension. Curr Opin Nephrol Hypertens. 2004;13:359-64.

11. Budhiraja R, Sharief I, Quan SF. Sleep disordered breathing and hypertension. JCSM. 2005;1:401-4.

12. Lavie P, Herer P, Hoffstein V. Obstructive sleep apnoea syndrome as a risk factor for hypertension: population study. BMJ. 2000;320:479-82.

13. Kiely JL, McNicholas WT. Cardiovascular risk factors in patients with obstructive sleep apnoea syndrome. Eur Respir J. 2000;16:128-33.

14. Bassetti CL, Milanova M, Gugger M. Sleep-disordered breathing and acute ischemic stroke - Diagnosis, risk factors, treatment, evolution, and long-term clinical outcome. Stroke. 2006;37:967-72.
15. Arzt M, Young T, Finn L, Skatrud JB, Bradley TD. Association of sleep-disordered breathing and the occurrence of stroke. Am J Respir Crit Care Med. 2005;172:1447-51.

16. Munoz R, Duran-Cantolla J, Martinez-Vila E, Gallego J, Rubio R, Aizpuru F, et al. Severe sleep apnea and risk of ischemic stroke in the elderly. Stroke. 2006;37:2317-21.

17. George CFP. Sleep - 5: Driving and automobile crashes in patients with obstructive sleep apnoea/hypopnoea syndrome. Thorax. 2004;59:804-7.

18. Lyznicki JM, Doege TC, Davis RM, Williams MA. Sleepiness, driving, and motor vehicle crashes. JAMA. 1998;279:1908-13.

19. Horne J, Reyner L. Vehicle accidents related to sleep: a review. Occup Environ Med. 1999;56:289-94.

20. Marin JM, Carrizo SJ, Vicente E, Agusti AG. Long-term cardiovascular outcomes in men with obstructive sleep apnoeahypopnoea with or without treatment with continuous positive airway pressure: an observational study. Lancet. 2005;365:1046-53.

21. Campos-Rodriguez F, Peña-Griñan N, Reyes-Nuñez N, De la Cruz-Moron I, Perez-Ronchel J, De la Vega-Gallardo F, et al. Mortality in obstructive sleep apnea-hypopnea patients treated with positive airway pressure. Chest. 2005;128:624-33.

22. Marti S, Sampol G, Munoz X, Torres F, Roca A, Lloberes $\mathrm{P}$, et al. Mortality in severe sleep apnoea/hypopnoea syndrome patients: impact of treatment. Eur Respir J. 2002;20(6):1511-8.

23. Yaggi HK, Concato J, Kernan WN, Lichtman JH, Brass LM, Mohsenin V. Obstructive sleep apnea as a risk factor for stroke and death. N Engl J Med. 2005;353:2034-41.

24. Bixler EO, Vgontzas AN, Lin HM, Ten Have T, Rein J, Vela-Bueno A, et al. Prevalence of sleep-disordered breathing in women: effects of gender. Am J Respir Crit Care Med. 2001;163:608-13.

25. Gislason T, Benediktsdottir B, Bjornsson JK, Kjartansson G, Kjeld M, Kristbjarnarson H. Snoring, hypertension, and the sleep apnea syndrome. An epidemiologic survey of middle-aged women. Chest. 1993;103:1147-51.

26. Durán J, Esnaola S, Ramón R, Iztueta A. Obstructive sleep apnea-hypopnea and related clinical features in a populationbased sample of subjects aged 30 to 70 years. Am J Respir Crit Care Med. 2001;163:685-9.

27. Young T, Palta M, Dempsey J, Skatrud J, Weber S, Badr $\mathrm{S}$. The occurrence of sleep-disordered breathing among middle-aged adults. N Eng J Med. 1993;328:1230-5.

28. Durán J, Esnaola S, Rubio R, De la Torre G. Obstructive sleep apnoea-hypopnoea in the elderly. A population-based study in the general population aged 71-100. Annual Meeting of ERS - Madrid. European Respiratory J. 2000;16:167S.

29. Caples SM, Gami AS, Somers VK. Obstructive sleep apnea. Ann Intern Med. 2005;142:187-97.

30. Stradling JR, Davies RJO. Sleep - 1: Obstructive sleep apnoea/ hypopnoea syndrome: definitions, epidemiology, and natural history. Thorax. 2004;59:73-8.

31. Guilleminault C, Bassiri A. Clinical features and evaluation of obstructive sleep apnea-hypopnea syndrome and upper 
airway resistance syndrome. En: Principles and Practice of Sleep Medicine, Fourth Edition. Philadelphia: Elsevier Saunders. 2005: 1043-52.

32. Puertas FJ, Pin-Arboledas G, Santa María-Cano J, DuránCantolla J. Consenso Nacional sobre el síndrome de apneashipopneas del sueño (SAHS). Grupo Español de Sueño. Arch Bronconeumol. 2005;41:72-9.

33. Ram CVS. Management of refractory hypertension. Am J Ther. 2003;10:122-6.

34. Cormican LJ, Williams A. Sleep disordered breathing and its treatment in congestive heart failure. Heart. 2005;91:1265-70.

35. Bailey DR, Attanasio R. Dentistry's role in the management of sleep disorders. Recognition and management. Dent Clin North Am. 2001;45:619-30.

36. Gordon P, Sanders MH. Sleep - 7: Positive airway pressure therapy for obstructive sleep apnoea/hypopnoea síndrome. Thorax. 2005;60:68-75.

37. Ryan CF. Sleep - 9: An approach to treatment of obstructive sleep apnoea/hypopnoea syndrome including upper airway surgery. Thorax. 2005;60:595-604.

38. Moyera CA, Sonnada SS, Garetzc SL, Helmand JI, Chervin RD. Quality of life in obstructive sleep apnea: a systematic review of the literature. Sleep Medicine. 2001;2:477-91.

39. Rey de Castro J. Perspectivas actuales en el tratamiento del Síndrome de Apneas-Hipopneas del Sueño (SAHS) y dificultades para su implementación en nuestro medio. Rev Med Hered. 2002;13:64-73.

40. Flemons WW, Douglas NJ, Kuna ST, Rodenstein DO, Wheatley J. Access to diagnosis and treatment of patients with suspected sleep apnea. Am J Respir Crit Care Med. 2004;169:668-72.

41. Kapur V, Blough DK, Sandblom RE, Hert R, de Maine JB, Sullivan SD, et al. The medical cost of undiagnosed sleep apnea. Sleep. 1999;22:749-55.

42. Ayas NT, FitzGerald JM, Fleetham JA, White DP, Schulzer M, Ryan CF, et al. Cost-effectiveness of continuous positive airway pressure therapy for moderate to severe obstructive sleep apnea/hypopnea. Arch Intern Med. 2006;166:977-84.

43. Young T, Peppard PE, Gottlieb DJ. Epidemiology of obstructive sleep apnea: a population health perspective. Am J Respir Crit Care Med. 2002;165:1217-39.

44. Peppard PE, Young T, Palta M, Dempsey J, Skatrud J. Longitudinal study of moderate weight change and sleepdisordered breathing. JAMA. 2000;284:3015-21.

45. Oksenberg A, Silverberg DS, Arons E, Radwan H. Positional vs nonpositional obstructive sleep apnea patients: anthropomorphic, nocturnal polysomnographic, and multiple sleep latency test data. Chest. 1997;112:629-39.

46. Young T, Finn L, Austin D, Peterson A. Menopausal status and sleep-disordered breathing in the Wisconsin Sleep Cohort Study. Am J Respir Crit Care Med. 2003;167:1181-5.
47. Schlosshan D, Elliott MW. Sleep - 3: Clinical presentation and diagnosis of the obstructive sleep apnoea hypopnoea syndrome. Thorax. 2004;59:347-52.

48. Teculescu D, Benamghar L, Hannhart B, Michaely JP. Habitual loud snoring. A study of prevalence and associations in 850 middle-aged French males. Respiration. 2006;73:68-72.

49. O'Connor GT, Lind BK, Lee ET, Nieto FJ, Redline S, Samet JM, et al. Variation in symptoms of sleep-disordered breathing with race and ethnicity: the Sleep Heart Health Study. Sleep. 2003;26:74-9.

50. Rey de Castro J, Álvarez J, Gaffo A. Síntomas relacionados a trastornos del sueño en supuestos sanos que asisten a un centro de Atención Primaria de Salud. Rev Med Hered. 2005;16:31-8.

51. Endeshaw Y. Clinical characteristics of obstructive sleep apnea in community-dwelling older adults. J Am Geriatr Soc. 2006;54:1740-4.

52. Valipour A, Lothaller H, Rauscher H, Zwick H, Burghuber OC, Lavie P. Gender-related differences in symptoms of patients with suspected breathing disorders in sleep: a clinical population study using the sleep disorders questionnaire. Sleep. 2007;30:312-9.

53. Young T, Evans L, Finn L, Palta M. Estimation of the clinically diagnosed proportion of sleep apnea syndrome in middle-aged men and women. Sleep. 1997;20:705-6.

54. Lechky O. Questions about sleep should be routine part of patient visits, physician says. CMAJ. 1993;149:1296-8.

55. Kushida CA, Littner MR, Morgenthaler T, Alessi CA, Bailey $\mathrm{D}$, Coleman J Jr, et al. Practice parameters for the indications for polysomnography and related procedures: An update for 2005. Sleep. 2005;28:499-521.

56. Rey de Castro J, Vizcarra D. Pautas de trabajo clínico, estándares de referencia, indicaciones de procedimientos y tratamiento en la medicina del sueño. Propuesta luego de tres años de actividad en un centro especializado en Lima. Rev Soc Per Med Interna. 1999;12:110-3.

57. Rey de Castro J, Tagle I, Escalante P. Síndrome ApneaHipopnea Obstructiva del Sueño (SAHOS). Propuesta para su diagnóstico y tratamiento. Comité Apnea Sueño de la Sociedad Peruana de Neumología. Rev Soc Per Neumología. 2001;44:24-8.

Manuscrito recibido el 06 de marzo de 2007 y aceptado para publicación el 06 de marzo de 2007.

\section{Correspondencia:}

Edmundo Rosales Mayor

Fidel Olivas Escudero 151 Dpto 301, San Miguel.

Lima 32, Perú

Correo-e:08251@upch.edu.pe 